Revue d'histoire de l'Amérique française

ZAS REVUE D.HISTOIRE DE L'AMÉRIQUE FRANÇAISE

\title{
À propos de la mise en nourrice à Québec pendant le Régime français
}

\section{Danielle Gauvreau}

Volume 41, numéro 1, été 1987

URI : https://id.erudit.org/iderudit/304522ar

DOI : https://doi.org/10.7202/304522ar

Aller au sommaire du numéro

Éditeur(s)

Institut d'histoire de l'Amérique française

ISSN

0035-2357 (imprimé)

1492-1383 (numérique)

Découvrir la revue

Citer cette note

Gauvreau, D. (1987). À propos de la mise en nourrice à Québec pendant le Régime français. Revue d'histoire de l'Amérique française, 41(1), 53-61. https://doi.org/10.7202/304522ar
Résumé de l'article

La mise en nourrice est un phénomène très répandu en France pendant l'Ancien Régime. Après une revue des principales caractéristiques de cette pratique en Europe, cette note de recherche s'interroge sur son existence au Canada pendant le Régime français. Il ressort de données relatives à la ville de Québec avant 1730 que le phénomène existe bel et bien, qu'il tend à augmenter avec le temps et qu'il touche surtout les familles des catégories supérieures de l'échelle professionnelle. 
NOTE DE RECHERCHE

\title{
A PROPOS DE LA MISE EN NOURRICE A QUÉBEC PENDANT LE RÉGIME FRANÇAIS
}

\author{
DANIELLE GAUVREAU \\ SOREP \\ Université du Québec à Chicoutimi
}

\section{RÉSUMÉ}

La mise en nourrice est un phénomène très répandu en France pendant l'Ancien Régime. Après une revue des principales caractéristiques de cette pratique en Europe, cette note de recherche s'interroge sur son existence au Canada pendant le Régime français. Il ressort de données relatives à la ville de Québec avant 1730 que le phénomène existe bel et bien, qu'il tend à augmenter avec le temps et qu'il touche surtout les familles des catégories supérieures de l'échelle professionnelle.

\section{ABSTRACT}

Wetnursing is a common phenomenon in Ancien Régime France. This text reviews the main features of the phenomenon in Europe and demonstrates its existence in Canada during the French Régime. Data from Quebec City before 1730 indicate the extent of the practice; they show that it increased over time and was found mainly among the upper echelons of the socio-professional structure.

Pratique très répandue en France pendant l'Ancien Régime, la mise en nourrice fut-elle pratiquée au Canada pendant le Régime français? La question est importante, d'une part parce que le phénomène comporte généralement des conséquences démographiques non négligeables (sur les niveaux de fécondité et de mortalité infantile) et d'autre part parce qu'il nous éclaire sur un aspect très concret de la vie des femmes et des familles à cette époque.

Après un rappel des principales caractéristiques du phénomène en Europe, nous rassemblons dans cette note de recherche divers éléments d'information qui permettent de répondre à la question initiale. Les résultats de nature quantitative concernent la ville de Québec avant 1730 et sont tirés de la thèse de doctorat que nous avons soutenue l'an dernier en démographie: ils figurent dans un chapitre portant sur le destin des enfants, où nous avons été amenée à apprécier l'ampleur de 
la mise en nourrice dans la ville de Québec suivant la catégorie professionnelle du père ${ }^{1}$.

\section{A - UN APERÇU DE LA PRATIQUE EN EUROPE}

En Europe, et surtout en France, la mise en nourrice constitue une pratique largement répandue durant l'Ancien Régime, particulièrement dans les milieux urbains. Dès sa naissance, l'enfant est envoyé à la campagne chez la nourrice qui a été choisie pour lui et il y reste durant les premières années de sa vie. Plus rarement, et dans les milieux aisés seulement, la nourrice vient vivre sous le toit familial pour y remplir ses fonctions.

Le recours à des nourrices soulage les femmes de leurs obligations maternelles traditionnelles. Non seulement celles-ci n'ont plus à allaiter leur enfant, mais le plus souvent l'enfant disparaît complètement, et pour une période relativement longue, des préoccupations quotidiennes du couple. Il semble bien que cette situation soit à l'origine de la popularité de cette coutume ${ }^{2}$. Dans les milieux populaires d'abord, la nécessité économique du travail des mères s'accommode mal de tâches maternelles particulièrement lourdes du fait que l'enfant est sevré assez tard. La mise en nourrice apparaît alors nécessaire à la vie économique du ménage.

Ce n'est certes pas le cas dans les autres milieux, où les considérations invoquées en faveur de cette pratique ont plutôt trait à un souci esthétique, à la crainte de l'épuisement, ou encore à l'incompatibilité perçue entre allaitement et vie sexuelle. L'idée générale que l'air sain de la campagne est favorable aux enfants et l'habitude bien établie de la mise en nourrice achèvent de faire de cette pratique une coutume allant de soi. Son existence tient évidemment aussi à la présence de femmes de la campagne disposées à offrir leurs services de nourrice contre une rémunération dont elles ont souvent un besoin pressant.

L'origine de ce phénomène n'est pas bien connue, mais il semble qu'il s'agisse dès le milieu du $17 \mathrm{e}$ siècle d'une pratique bien ancrée dans les moeurs ${ }^{3}$. Si elle touche au départ davantage les milieux aristocratiques, puis la bourgeoisie, elle n'en gagne pas moins au cours du 18 e siècle l'ensemble des couches de la société urbaine. Les exemples

\footnotetext{
1 Danielle Gauvreau, «Reproduction humaine et reproduction sociale: la ville de Québec pendant le Régime français», thèse de Ph.D. (démographie), Université de Montréal, 1986.

2 Pour un rappel des raisons invoquées en faveur de la mise en nourrice, voir par exemple Marie-France Morel, «Théories et pratiques de l'allaitement en France au XVIIIe siècle», Annales de démographie historique (1976): 393-427.

${ }_{3}$ Jean-Pierre Bardet, Rouen aux XVIIe et XVIIIe siècles. Les mutations d' un espace social (Paris, Société d'édition d'enseignement supérieur, 1983), 303.
} 
sont nombreux: Paris, d'abord, où l'organisation de cette pratique fait appel à un bureau des nourrices, Rouen, Genève, Lyon, Meulan, et même la petite châtellenie de Thoissey-en-Dombes étudiée par Bideau ${ }^{4}$. Surtout répandue en France, elle touche probablement aussi dans une moindre mesure d'autres régions d'Europe.

L'ampleur exacte de la mise en nourrice n'est pas facile à établir et varie suivant plusieurs variables. A Genève, Perrenoud fait état de $10 \%$ d'enfants morts en nourrice parmi les décès d'enfants de 0 à 11 mois entre 1730 et 1739 , puis de $31 \%$ entre 1775 et $1784^{5}$. Pour la période allant de 1740 à 1789, Bardet obtient pour Rouen les proportions suivantes d'enfants mis en nourrice selon le statut social du père: notables, $71 \%$; boutiquiers, 64\%; artisans, $51 \%$; ouvriers, $41 \%{ }^{6}$. Dans l'ensemble, Badinter estime que les Françaises eurent recours si massivement à cette pratique «qu'au milieu du XVIIIe siècle, on considère que les enfants des villes allaités par leurs mères étaient des exceptions» ${ }^{7}$.

Un phénomène d'une telle ampleur n'est pas sans avoir des conséquences démographiques importantes, souvent liées aux conditions de son exercice. Flandrin les résume bien en parlant de la surfécondité des couples urbains et de la surmortalité des enfants ${ }^{8}$, illustrée également par des études telles celles de Bardet sur Rouen. Le premier aspect résulte directement de l'absence d'allaitement maternel qui, comme on le sait, s'accompagne d'une période d'aménorrhée post partum. Sans empêcher complètement toute conception, l'allaitement n'en réduit pas moins considérablement les risques de grossesse et, en son absence, les intervalles qui séparent deux naissances apparaissent nettement moins importants; cet effet sur le calendrier des naissances se traduit par une augmentation des niveaux de fécondité.

La mortalité infantile, déjà non négligeable à l'époque étudiée, paraît quant à elle encore augmentée par la pratique de mise en nourrice. Dès le départ, le transport des nourrissons de la ville à la campagne est

\footnotetext{
4 Voir à cet effet Marcel Lachiver, «Nourrices et nourrissons en région parisienne au XVIIIe siècle», Histoire et archéologie dans les Yvelines, supplément à Connaître les Yvelines (Versailles, 1977), 14-22; La population de Meulan du XVIIe au XIXe siècle (vers 1600-1870). Étude de démographie historique (Paris, SEVPEN, 1969), 339 p.; Jean-Pierre Bardet, op. cit.; Alfred Perrenoud, La population de Genève du XVIe au début du XIXe siècle: étude démographique (Paris, H. Champion, Genève, A. Julien, 1979), volume 1; Maurice Garden, Lyon et les Lyonnais au XVIIle siècle (Paris, Flammarion, 1975), 374 p.; Alain Bideau, «L'envoi des jeunes enfants en nourrice. L'exemple d'une petite ville: Thoissey-en-Dombes (1740-1840)», Sur la population française au XVIIIe et au XIXe siècles: Hommage à Marcel Reinard (Paris, 1973), 49-58.

5 A. Perrenoud, op. cit., 415.

6 J.-P. Bardet, op. cit., 302.

7 Elisabeth Badinter, L'amour en plus: histoire de l'amour maternel (XVIIe-XXe siècle) (Paris, Flammarion, 1980), 89-90.

8 Jean-Louis Flandrin, Familles, parenté, maison, sexualité dans l'ancienne société (Paris, Hachette, 1976), 287 p.
} 
fatal à plusieurs d'entre eux qui sont plus fragiles, ou tout simplement victimes d'accidents, de négligences. Par la suite, les conditions de pauvreté, de malpropreté, de manque de soins et de nourriture insuffisante dans lesquelles doivent vivre plusieurs enfants sont la source de décès nombreux ${ }^{9}$. La mortalité infantile apparaît nettement plus importante parmi les enfants placés en nourrice: Bardet en fait état pour la ville de Rouen ${ }^{10}$, tout comme Flandrin et Shorter qui parlent d'une mortalité deux fois plus élevée parmi ce groupe d'enfants. Cette conséquence dévastatrice du phénomène est d'ailleurs probablement à l'origine de sa contestation à partir de la seconde moitié du $18 \mathrm{e}$ siècle.

Étudiant le développement d'un discours spécifique sur l'enfant, Morel relie en effet le développement d'un discours en faveur de l'allaitement maternel et son corollaire, le développement d'une opposition à la mise en nourrice, à des visées populationnistes tendant à contrer les effets d'une mortalité infantile surélevée ${ }^{11}$. Il s'agit là d'un argument politique auquel s'ajoutent des considérations médicales et morales. Pour Badinter, l'abandon progressif de cette pratique coïncide avec la naissance de l'amour maternel; Shorter l'associe pour sa part à la naissance de la famille «moderne», tandis que les thèses d'Ariès sur l'enfant et la vie familiale y associeraient plutôt la naissance de l'enfance et de sentiments jusqu'alors inconnus à l'égard de l'enfant ${ }^{12}$. Chacun à leur façon, ces auteurs font état d'éléments qui se conjuguent en fait pour miner progressivement les fondements d'une pratique pourtant considérée comme normale dans plusieurs milieux. Sans être spectaculaire, son abandon n'en paraît pas moins sensible à certains observateurs vers la fin du $18 \mathrm{e}$ siècle ${ }^{13}$.

\section{B - LA MISE EN NOURRICE A QUÉBEC PENDANT LE RÉGIME FRANÇAIS}

\section{1 - La situation au Canada}

La pratique de la mise en nourrice s'est-elle transportée au Canada au moment de la colonisation?

Étant assez généralisée en France à cette époque, on peut difficilement imaginer qu'elle n'ait pas été introduite au Canada, surtout dans

\footnotetext{
y Voir à cet effet la description de certaines conditions de vie chez les nourrices telles que rapportées par Edward Shorter, Naissance de la famille moderne, XVIIle-XXe siècle (Paris, Éditions du Seuil, 1975), 222-225.

10 J.-P. Bardet, op. cit., 369.

11 M.-F. Morel, op. cit.

12 Voir Badinter, op. cit.; Shorter, op. cit., ainsi que Philippe Ariès, L'enfant et la vie familiale sous l'Angien Régime (Paris, Éditions du Seuil, 1973), 316 p.

13 Voir Shorter, op. cit. et Morel, op. cit. Les données rassemblées par Bardet (Rouen) et Perrenoud (Genève) suggèrent toutefois une généralisation du phénomène au cours du $18 \mathrm{e}$ siècle et même au début du $19 \mathrm{e}$ siècle.
} 
les milieux aristocratiques et bourgeois. Ceci, d'une part parce que ces groupes sont davantage touchés par le phénomène au 17e siècle et, d'autre part, parce qu'ils proviennent probablement plus souvent de milieux urbains où la pratique est plus répandue. Les villes étant par ailleurs encore peu développées au Canada, la pression économique en faveur de la mise en nourrice pour les femmes des milieux populaires se fait probablement moins vive.

En l'absence d'étude statistique du phénomène, un certain nombre d'indices témoignent cependant de son existence tout au long du Régime français, et ce non seulement dans les cas où la mère décède rapidement et où la mise en nourrice s'impose alors. Denise Lemieux les a rassemblés dans son ouvrage sur l'enfance en Nouvelle-France ${ }^{14}$. Elle cite ainsi une mise en garde de Mgr de Saint-Vallier de même qu'une ordonnance de l'intendant Bégon à ce propos. Cette pratique est par ailleurs attestée par une étude de Joseph-Edmond Roy, qui identifie les enfants morts en nourrice à la Pointe-Lévis, au cours des 50 dernières années du Régime français ${ }^{15}$. Plusieurs de ces enfants paraissent d'origine sociale élevée; ce trait se trouve renforcé par une autre étude portant sur la famille d'Ailleboust, qui a également recours à cette pratique ${ }^{16}$. Enfin, quelques procès font clairement référence à des situations de mise en nourrice où l'entente n'aurait pas été respectée.

Quoique partiels, les éléments précédents, ajoutés à ce que nous savons déjà de la pratique de la mise en nourrice en France à la même époque, paraissent suffisants pour penser que le phénomène a bel et bien existé au Canada. Suivant quelle ampleur et dans quels milieux? C'est ce que nous cherchons maintenant à préciser pour la ville de Québec avant 1730.

\section{2 - La mise en nourrice à Québec avant 1730}

L'identification des enfants placés en nourrice s'est faite à partir de l'utilisation conjointe des familles reconstituées de la ville de Québec avant 1765 , déjà utilisées dans notre thèse, et des familles reconstituées par le Programme de recherche en démographie historique du département de démographie de l'Université de Montréal (PRDH) pour l'ensemble de la colonie jusqu'en 1730. Ainsi, toutes les familles dont le mariage a été célébré à Québec et qui comptent des enfants nés dans cette ville mais décédés dans une autre paroisse alors qu'ils étaient âgés de moins de deux ans, ont été identifiées dans la reconstitution du PRDH.

\footnotetext{
14 Denise Lemieux, Les petits innocents. L'enfance en Nouvelle-France (Québec, Institut québécois de recherche sur la culture, 1985), 205 p.

${ }_{15}$ Joseph-Edmond Roy, Histoire de la seigneurie de Lauzon (Lévis, Mercier et Cie, 1897. 1900), 2: 190. (Étude citée par Denise Lemieux).

16 Lorraine Gadoury, «Une famille noble en Nouvelle-France: les d'Ailleboust», mémoire de maîtrise (histoire), Université de Montréal, 1982. (Étude rapportée par Denise Lemieux).
} 
Après une première vérification qui visait à éliminer les familles qui s'étaient vraisemblablement installées dans une paroisse autre que Québec après la naissance de l'enfant, nous avons associé les décès restants à des décès d'enfants morts en nourrice ${ }^{17}$. Une telle association ne va pas sans risque, puisque seulement une petite minorité des actes concernés mentionnent que l'enfant décédé se trouvait en nourrice dans la paroisse (il y a 5 inscriptions de ce type); cependant, la moitié des actes font clairement état d'un lieu de résidence à Québec pour les parents, alors que les autres ne contiennent le plus souvent aucune mention de résidence. Par ailleurs, la concentration de ces décès dans certaines familles (53\% des décès proviennent de familles où l'on en compte plus d'un) et leur provenance très nette des paroisses voisines de Québec renforcent l'idée qu'il s'agit bien de cas de mise en nourrice. Nous les avons donc considérés comme tels et les avons analysés pour dégager les caractéristiques des familles qui plaçaient leurs enfants en nourrice.

Suivant cette analyse, la mise en nourrice existe bel et bien dans la ville de Québec sous le Régime français: il s'agit d'un phénomène de faible ampleur, qui s'intensifie toutefois avec le temps et touche plus particulièrement certains groupes professionnels. Seulement $6 \%$ des décès observés sont survenus après le décès de la mère, lequel a alors pu constituer un facteur important dans la décision de placer l'enfant en nourrice. Aucun cas de décès présumé d'enfant en nourrice n'apparaît avant 1680; 17 ont lieu entre 1680 et 1700 , puis 86 entre 1700 et 1720 et 62 entre 1720 et 1730, une période de dix ans seulement. Les paroisses où sont enregistrés ces décès sont clairement les paroisses rurales des environs de la ville: Charlesbourg, surtout, qui compte près de la moitié des décès, puis Beauport et l'Ancienne-Lorette; ensemble, les trois paroisses regroupent plus de $75 \%$ de tous les décès (tableau 1).

Par rapport au nombre total d'enfants nés au cours de ces mêmes périodes dans les familles dont la fiche s'ouvre par un mariage, cela représente à partir de 1680 des proportions respectives de $1,4 \%, 5,1 \%$ et $4,3 \%$. Évidemment, tous les enfants ne mouraient pas en nourrice, de sorte que ces proportions ne rendent compte que d'une évolution

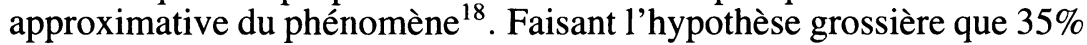
des enfants mouraient à cette époque avant leur second anniversaire, nous évaluons à près de $4 \%$ la proportion d'enfants placés en nourrice

17 Nous ne saisissons probablement là qu'une partie des enfants morts en nourrice, ceux placés dans les paroisses voisines de Québec, mais c'est probablement la majorité des cas si le phénomène suit, comme il est permis de le croire, le modèle européen.

18 La baisse au cours de la dernière période n'est pas significative, d'autant moins que certains décès d'enfants nés peu avant 1730 ont pu nous échapper. 
TABLEAU 1

Enfants nés à Québec entre 1680 et 1730 et décédés à moins de 2 ans à l'extérieur de Québec, selon la paroisse du décès et la période de naissance.

\begin{tabular}{|c|c|c|c|c|c|c|c|c|}
\hline \multirow{3}{*}{$\begin{array}{l}\text { Paroisse } \\
\text { du décès }\end{array}$} & \multicolumn{8}{|c|}{ Période } \\
\hline & \multicolumn{2}{|c|}{$1680-1699$} & \multicolumn{2}{|c|}{$1700-1719$} & \multicolumn{2}{|c|}{$1720-1729$} & \multicolumn{2}{|c|}{ Ensemble } \\
\hline & $\mathrm{N}$ & $\%$ & $\mathrm{~N}$ & $\%$ & $\mathrm{~N}$ & $\%$ & $\mathrm{~N}$ & $\%$ \\
\hline Charlesbourg & 3 & 17,6 & 48 & 55,8 & 29 & 46,8 & 80 & 48,5 \\
\hline Beauport & 7 & 41,2 & 13 & 15,1 & 9 & 14,5 & 29 & 17,6 \\
\hline Ancienne-Lorette & 1 & 5,9 & 12 & 14,0 & 5 & 8,1 & 18 & 10,9 \\
\hline Saint-Augustin & 2 & 11,8 & 3 & 3,4 & 6 & 9,7 & 11 & 6,7 \\
\hline Lévis & - & - & 1 & 1,2 & 8 & 12,9 & 9 & 5,5 \\
\hline Sainte-Foy & - & - & 2 & 2,3 & 2 & 3,2 & 4 & 2,4 \\
\hline Neuville & 4 & 23,5 & - & - & 1 & 1,6 & 5 & 3,0 \\
\hline Beaumont & - & - & 3 & 3,4 & 1 & 1,6 & 4 & 2,4 \\
\hline $\begin{array}{l}\text { Saint-Laurent } \\
\text { (île d'Orléans) }\end{array}$ & - & - & 1 & 1,2 & 1 & 1,6 & 2 & 1,2 \\
\hline Saint-Michel & - & - & 1 & 1,2 & - & - & 1 & 0,6 \\
\hline Saint-Nicholas & - & - & 1 & 1,2 & - & - & 1 & 0,6 \\
\hline Montréal & - & - & 1 & 1,2 & - & - & 1 & 0,6 \\
\hline Ensemble & 17 & 100 & 86 & 100 & 62 & 100 & 165 & 100 \\
\hline
\end{tabular}

Source: Programme de recherche en démographie historique: familles reconstituées à partir des registres des paroisses de l'ensemble de la colonie de 1621 à 1730.

entre 1680 et 1700 , et à près de $15 \%$ après cette date ${ }^{19}$. Ce niveau se maintient probablement après 1730 et il est même possible qu'il continue d'augmenter. Il ne parait toutefois pas suffisamment important pour avoir influencé de façon significative les niveaux de fécondité en comparaison avec des régions où la mise en nourrice n'était pas pratiquée.

A Québec, tous les groupes professionnels ne placent pas leurs enfants en nourrice. Par rapport à celle des enfants nés à Québec entre 1680 et 1730, la répartition des enfants décédés ailleurs qu'à Québec fait voir une nette sur-représentation de la progéniture des officiers militaires et civils surtout, ainsi que de celle des marchands (tableau 2). A l'opposé, les familles des artisans de la construction paraissent les moins nombreuses à avoir recours à cette pratique. Les résultats du tableau 2 se trouvent par ailleurs confirmés par la distribution des intervalles entre

\footnotetext{
19 Il est difficile de savoir si, dans la colonie, les enfants placés en nourrice mouraient davantage que les autres: c'est là une situation largement répandue en Europe, mais il n'est pas certain qu'elle se vérifie également au Canada.
} 
TABLEAU 2

Enfants nés à Québec et décédés à moins de deux ans entre 1680 et 1730, selon la catégorie professionnelle du père, parmi les familles dont le couple s'est marié à Québec.

\begin{tabular}{|c|c|c|c|c|}
\hline \multirow{3}{*}{ Catégorie professionnelle du père } & \multicolumn{4}{|c|}{ Enfants nés à Québec } \\
\hline & \multicolumn{2}{|c|}{ Total } & \multicolumn{2}{|c|}{$\begin{array}{l}\text { Dont décédés } \\
\text { à l'extérieur }\end{array}$} \\
\hline & $\mathrm{N}$ & $\%^{a}$ & $\mathrm{~N}$ & $\%^{\mathrm{a}}$ \\
\hline Officiers militaires et civils & 519 & 15,9 & 50 & 35,7 \\
\hline $\begin{array}{l}\text { Employés subalternes de } \\
\text { l'administration }\end{array}$ & 156 & 4,8 & 2 & 1,4 \\
\hline Soldats & 118 & 3,6 & 5 & 3,6 \\
\hline Marchands & 377 & 11,6 & 34 & 24,3 \\
\hline Artisans de la construction & 611 & 18,7 & 11 & 7,9 \\
\hline Artisans divers & 769 & 23,6 & 26 & 18,6 \\
\hline Navigateurs & 225 & 6,9 & 7 & 5,0 \\
\hline Charretiers & 65 & 2,0 & - & - \\
\hline Commerçants & 111 & 3,4 & 1 & 0,7 \\
\hline Journaliers & 74 & 2,3 & 2 & 1,4 \\
\hline Cultivateurs & 132 & 4,1 & - & - \\
\hline Divers & 101 & 3,1 & 2 & 1,4 \\
\hline Inconnu & 1117 & $(25,5)$ & 25 & $(15,2)$ \\
\hline Ensemble & 4375 & 100 & 165 & 100 \\
\hline
\end{tabular}

Source: Programme de recherche en démographie historique: familles reconstituées à partir des registres de la paroisse Notre-Dame-de-Québec de 1621 à 1765 et familles reconstituées à partir des registres des paroisses de l'ensemble de la colonie de 1621 à 1730 .

${ }^{a}$ Les proportions sont calculées sans les cas de profession inconnue, mais la part de ces derniers parmi l'ensemble figure entre parenthèses.

naissances. On observe en effet des différences à cet égard entre les familles d'artisans et de journaliers, d'une part, et celles des officiers et des marchands, d'autre part. Les familles de ces derniers présentant des intervalles nettement plus courts, on en déduit qu'elles avaient moins recours que les autres à l'allaitement maternel ${ }^{20}$.

\footnotetext{
20 Voir notre thèse à cet effet, à la page 341 et le tableau C. 16 de l'annexe statistique. Ces résultats sont d'autant plus reliés à des pratiques différentes d'allaitement maternel que les niveaux de mortalité infantile présentent, suivant les groupes professionnels, des différences qui agissent dans un sens opposé sur les intervalles entre naissances.
} 


\section{CONCLUSION}

Les résultats précédents montrent que le phénomène de la mise en nourrice existait bel et bien au Canada pendant le Régime français. Ce ne sont toutefois pas des raisons économiques qui paraissent surtout l'avoir motivée, puisque la pratique touche davantage les catégories aisées de l'échelle professionnelle. Il semble plus probable que le phénomène, largement répandu en Europe parmi ces groupes et dans les villes, soit tout simplement passé à son tour dans les moeurs d'une partie de la population coloniale, surtout au 18e siècle.

Comment étaient vues ces femmes, et plus généralement ces parents qui plaçaient leurs enfants en nourrice? Qui étaient ces femmes des paroisses rurales qui les prenaient en pension et dans quelles conditions vivaient-elles? Voilà de nouvelles questions auxquelles il faudra maintenant tenter de répondre tout en étendant l'observation à l'ensemble de la colonie et en poursuivant l'analyse sur une période plus longue. L'évolution ultérieure du phénomène et les circonstances entourant sa disparition demandent encore à être élucidées. 